\title{
The influence of some azoles on wound healing in albino rats
}

\author{
M.B. Girish, P.A.Patil
}

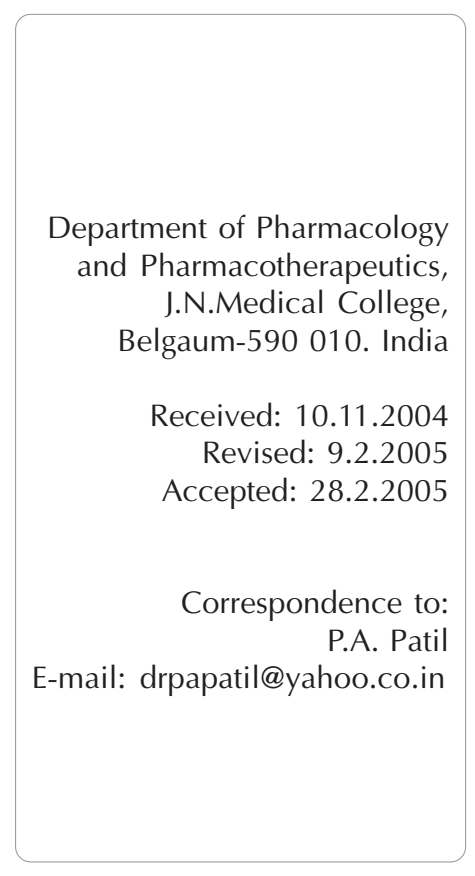

\begin{abstract}
Objective: To investigate the effect of azoles viz metronidazole (MTZ), tinidazole (TNZ), ketoconazole (KCZ), and fluconazole (FLZ) on resutured incision, excision, and dead space wounds in albino rats.

Materials and methods: Resutured incision, excision, and dead space wounds were inflicted in albino rats of either sex under light ether anesthesia, taking aseptic precautions. Control animals received vehicle, and other groups received MTZ, TNZ, KCZ, and FLZ orally for a period of 10 days in incision and dead space wounds, whereas in excision wounds till their complete closure. On the 11th day, after estimating breaking strength of resutured incision wounds (under anesthesia), animals were sacrificed and granulation tissue removed from dead space wounds to estimate breaking strength, hydroxyproline content, quantification of granulation tissue, and histological studies in control and treated groups. In the excision wound model, wound closure rate, epithelization time, and scar features were studied from the day of wounding till the day of scab falling off with no residual raw area.

Results: Except ketoconazole, rest of the azoles significantly $(\mathrm{P}<0.01,<0.05)$ promoted the healing process in all the three wound models studied.

Conclusion: Metronidazole, tinidazole and fluconazole promote wound healing, whereas ketoconazole does not do so.
\end{abstract}

KEYWORDS: Antiamebics, antifungals, wound models.

\section{Introduction}

Wound healing, a common clinical entity contemporary to human beings, has been recognized to be influenced by several factors such as nutrients like vit-C, zinc, and hormones like GH, insulin, etc. Products of traditionally used plants such as Bryophyllum pinnatum ${ }^{[1]}$ and Moringa oleifera ${ }^{[2]}$ have also been reported to promote healing in experimental animals, probably by virtue of their antimicrobial activity. Because it is well known that infection interferes with healing, antimicrobials are expected to suppress infection and promote healing process only in infected wounds. Antimicrobials are routinely administered pre- and post-surgically and metronidazole, a commonly used antiprotozoal for surgical chemoprophylaxis against anaerobes, is likely to influence the surgical outcome. However, metronidazole (MTZ) has been reported to delay the healing process in dead space wounds ${ }^{[3]}$ and reduce the breaking strength of facial wounds. ${ }^{[4]}$ Recently, MTZ has been reported to possess a potent anti-oxidant property that augments healing process in burns. ${ }^{[5]}$ Similarly, tinidazole (TNZ) has also been reported to suppress healing of dead space wounds, ${ }^{[3]}$ whereas fluconazole (FLZ), a related antifungal azole, has been shown to enhance epithelization. ${ }^{[13]}$ There are no reports on the influence of ketoconazole (KCZ) on wound healing, an antifungal related to fluconazole.
In view of the controversial reports about the effect of MTZ on healing and paucity of such information about other azoles, the present study was planned to investigate their influence on resutured incision, excision, and dead space wounds in albino rats.

\section{Materials and Methods}

\section{Animals and drug treatment}

Healthy male Wistar rats weighing (150-250 g) were housed individually on standard pellet diet with water ad libitum and were starved overnight before the day of experimentation. The study was approved by the institutional animal ethics committee. Depilation at wounding site was done a day before wounding. After wounding, the animals were divided into control and treatment groups ( $n=6$ in each) for each wound model to receive various treatments. MTZ (Unique), TNZ (Unichem), KCZ (Khandelwal) and FLZ (FDC), were obtained as generous gifts from the respective pharmaceutical companies. These drugs were administered orally in therapeutic equivalent doses (per kg body weight) as calculated with the help of the conversion table devised by 'Paget and Barnes'. ${ }^{[6]}$ The dose of MTZ (108 mg), TNZ (180 mg), KCZ (18 $\mathrm{mg}$ ), and FLZ (18 mg) were suspended in $2 \%$ gum acacia and were administered once daily in the volume of $5 \mathrm{~mL} / \mathrm{kg}$, while 
control groups received equal volume of the vehicle. The duration of treatment was 10 days for animals inflicted with incision and dead space wounds, whereas it was continued in animals bearing excision wounds till their complete closure. Wound models

1. Resutured incision wounds were inflicted with two $6 \mathrm{~cm}$ long paravertebral parallel incisions under light ether anesthesia as described earlier. ${ }^{[7]}$ Sutures were removed on the $7^{\text {th }}$ day; on the $10^{\text {th }}$ post wounding day, breaking strength was measured by the continuous water flow technique as described by 'Lee', ${ }^{,[8}$ and subsequently animals were sacrificed by overdose of anesthesia.

2. Excision wounds were made as described by 'Morton and Malone' ${ }^{[9]}$ by excising the full thickness circular skin (approximately $500 \mathrm{~mm}^{2}$ ) from the nape of neck under ether anesthesia. Wound-closure rate and epithelization time were assessed by tracing the wound on polythene paper from wounding day, followed by $4,8,12,16^{\text {th }}$ day and subsequently on alternate days till complete epithelization (fall of scab without any raw area). Similarly, scars were traced on complete epithelization to assess wound contraction by noting scar size and shape.

3. Dead space wounds were inflicted by implanting sterile cotton pellets $(10 \mathrm{mg})$ and cylindrical grass piths $(2.5 \mathrm{~cm}$ $\times 0.3 \mathrm{~cm})$ s.c. in the groin and axilla alternatively by the technique of D'Arcy et al. as described by Turner. ${ }^{[10]}$ On the $10^{\text {th }}$ post-wounding day, all the granulation tissues were removed under light ether anesthesia and dried at $60{ }^{\circ} \mathrm{C}$ overnight to record the dry weight which was expressed as mg per $100 \mathrm{~g}$, body weight as suggested by 'Dipasquale and Meli'. ${ }^{[1]}$ One of the granulation tissues over the grass piths was opened and trimmed to a rectangular piece for estimation of breaking strength and subsequently colorimetric estimation of hydroxyproline content as described earlier, ${ }^{[12]}$ whereas the other piece was preserved in $10 \%$ formalin for histological studies.

\section{Statistical analysis}

The data were analyzed using ANOVA followed by Dunnett's test. $\mathrm{P}$ values $<0.05$ were considered significant.

\section{Results}

The mean breaking strength of resutured incision wound and granulation tissue was significantly $(* \mathrm{P}<0.05)$ increased in all the drug-treated groups except in KCZ group in which there was marginal increase as compared to that of control group, but the difference was not statistically significant. The healing of dead space wounds as assessed by granulation tissue dry weight and hydroxyproline content of granuloma were also significantly $(* \mathrm{P}<0.05)$ promoted by all the drugs except KCZ. [Table1]

The histological studies of granulation tissues revealed increased number of fibroblasts and thick bundles of collagen tissue in MTZ, TNZ and FLZ-treated groups as compared to that of control and KCZ groups. [Figure 1a - 1e]

Excision wound-closure rate as compared to that of control was significantly $(* \mathrm{P}<0.05)$ increased throughout the study in MTZ, TNZ and FLZ groups, while it was so, only on the 12th and $16^{\text {th }}$ day in KCZ group. However, epithelization period was significantly $(* \mathrm{P}<0.05)$ decreased only in MTZ, TNZ and FLZtreated groups, as compared to that of the control group. There was significant reduction of scar area in MTZ and FLZ groups as compared to that of control, which did not significantly differ from those of TNZ or KCZ-treated groups. [Table 2] Scars were oval shaped with regular margins in control, TNZ and KCZtreated groups, whereas they were stellate shaped in MTZ and FLZ groups indicating enhanced wound contraction.

\section{Discussion}

Results of the present study clearly indicate that both MTZ and FLZ in their therapeutic equivalent doses promote the healing of all types of wounds studied and favorably altered

\section{Table 1}

Effect of azoles on resutured incision and dead space wound (Granulation tissue formation)

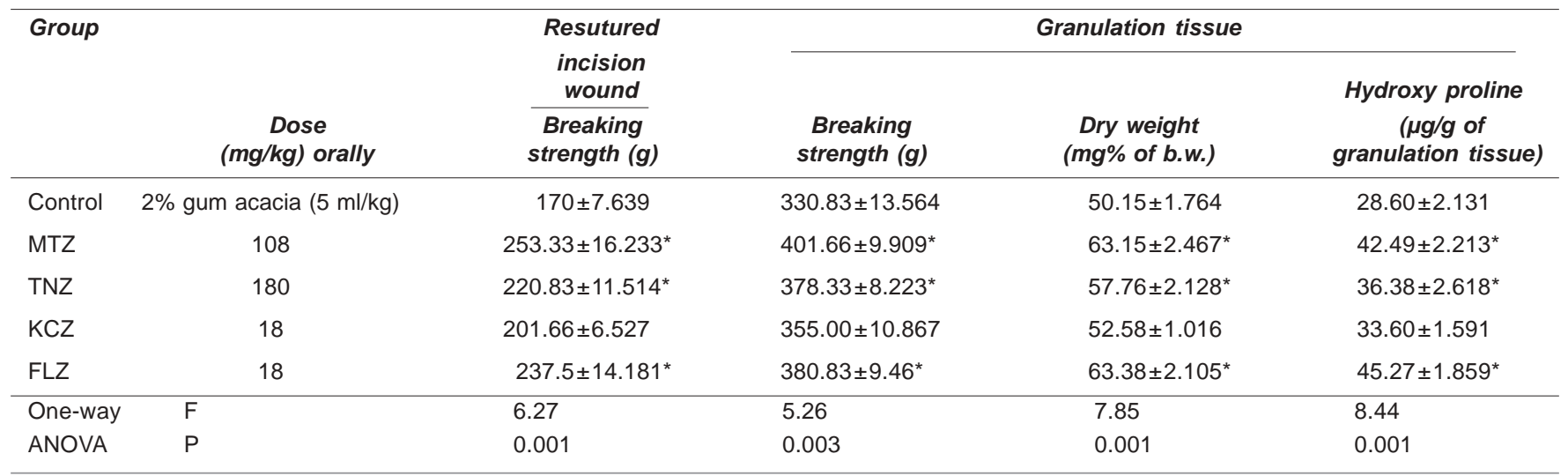

${ }^{*} \mathrm{P}<0.05$ as compared to control; $n=6$ in each group. $d f=4,25$. 
Table 2

Effect of azoles on excision wound model

\begin{tabular}{|c|c|c|c|c|c|c|c|c|}
\hline Group $(n=6)$ & $\begin{array}{c}\text { Dose } \\
\text { (mg/kg) } \\
\text { orally }\end{array}$ & \multicolumn{5}{|c|}{ Wound closure (\% of original area) in $\mathrm{mm}^{2}$ on day } & $\begin{array}{c}\text { Time for } \\
\text { epithelization } \\
\text { complete } \\
\text { (days) }\end{array}$ & $\begin{array}{l}\text { Scar } \\
\text { area } \\
\left(\mathrm{mm}^{2}\right)\end{array}$ \\
\hline MTZ & 108 & $29.1 \pm 2.67^{*}$ & $56.13 \pm 1.86^{*}$ & $78.08 \pm 1.15^{*}$ & $90.1 \pm 1.36^{*}$ & $98.3 \pm 0.94^{*}$ & $20.16 \pm 0.4^{*}$ & $33.66 \pm 0.608^{*}$ \\
\hline TNZ & 180 & $25.1 \pm 1.72^{*}$ & $51.6 \pm 1.28^{*}$ & $70.3 \pm 1.26^{*}$ & $85.5 \pm 1.33^{*}$ & $94.7 \pm 1.39^{*}$ & $22.33 \pm 0.6^{*}$ & $40.16 \pm 1.27$ \\
\hline FLZ & 18 & $27.01 \pm 1.4^{*}$ & $54.5 \pm 1.81^{*}$ & $73.8 \pm 1.46^{*}$ & $88.6 \pm 1.07^{*}$ & $97.1 \pm 1.18^{*}$ & $20.33 \pm 0.6^{*}$ & $35.5 \pm 0.69^{*}$ \\
\hline One-way & $\mathrm{F}$ & 7.22 & 12.85 & 34.52 & 22.19 & 12.79 & 7.78 & 14.33 \\
\hline ANOVA & $\mathrm{P}$ & $<0.01$ & $<0.01$ & $<0.01$ & $<0.01$ & $<0.01$ & $<0.01$ & $<0.01$ \\
\hline
\end{tabular}

Values are mean $\pm S E M ;{ }^{*} P<0.05$ as compared to control; $n=6$ in each group. $d f=4,25$.

Figure 1. Microphotographs of granulation tissue stained with H\&E (100 X).

1a. Control

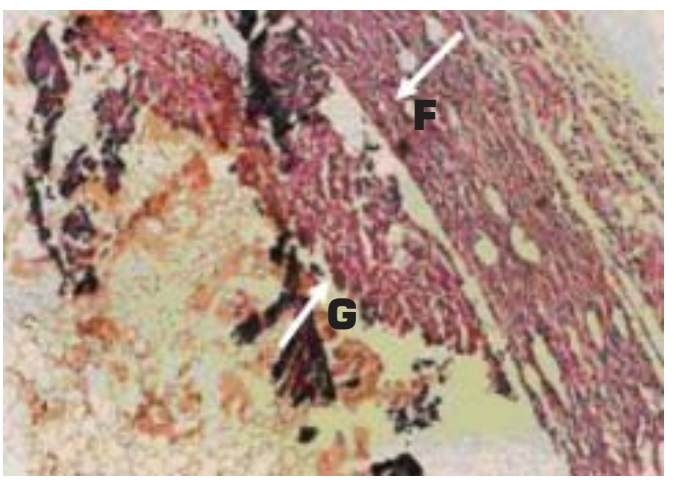

1c. Tinidazole

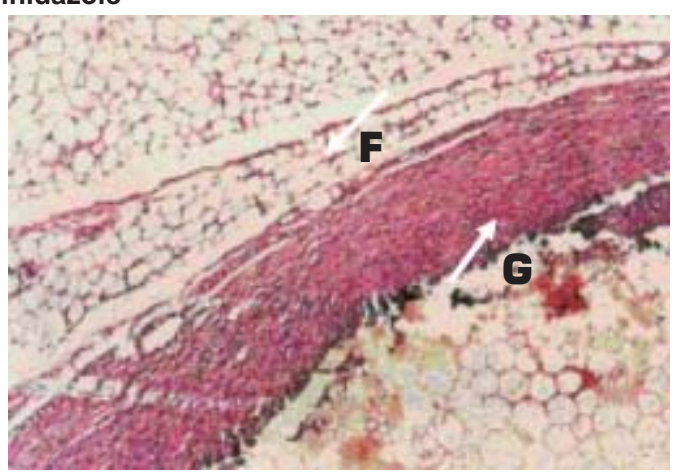

1e. Fluconazole

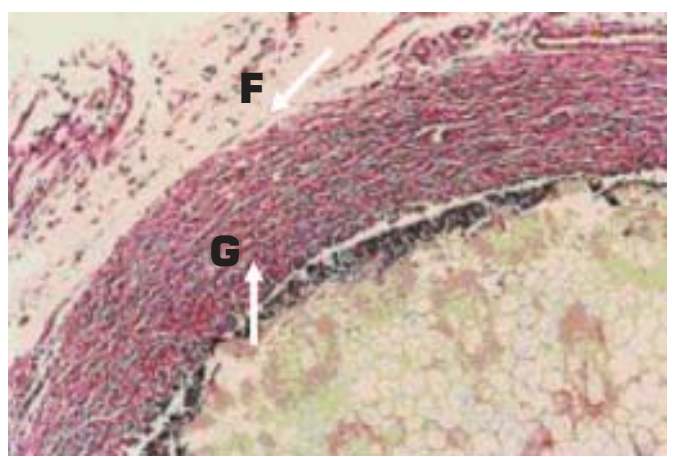

1b. Metronidazole

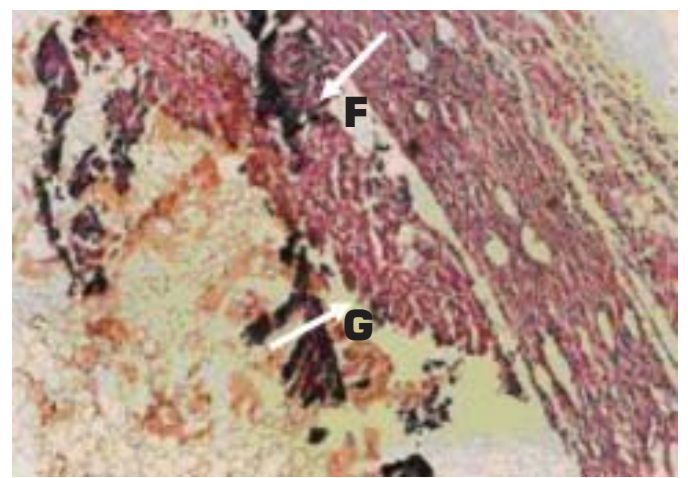

1d. Ketoconazole

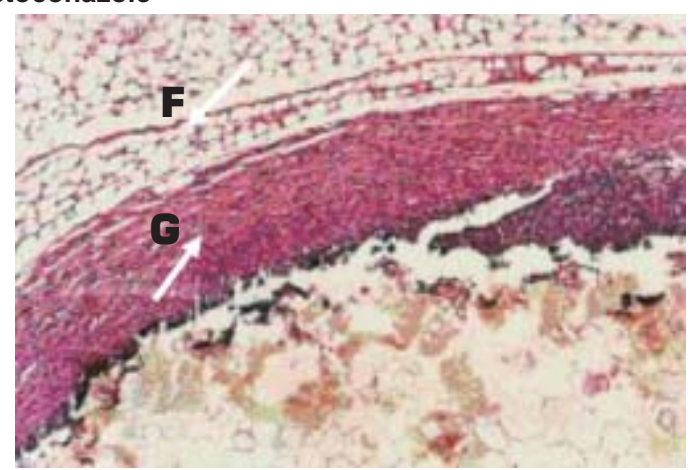

Note: Markedly increased fibrocollagen tissue (arrows) in MTZ, TNZ and FLZ treated groups as compared to that of $\mathrm{KCZ}$, which was almost comparable to that of control. G - Granulation tissue; F - Fibrocollagen tissue. 
the scar features. TNZ, though promoted healing in all the three wound models, it failed to reduce the scar size, whereas KCZ did not considerably alter the healing of dead space and resutured incision wounds but, promoted excision wound closure only at the 12 and $16^{\text {th }}$ days respectively.

The present findings of increased epithelization by MTZ and TNZ agree with the earlier report. ${ }^{[3]}$ Similarly, enhanced epithelization by FLZ as observed in the present study is in agreement with another previous study. ${ }^{[13]}$ Enhanced wound contraction in MTZ-treated animals of the present study corroborates with the earlier report ${ }^{[5]}$ in which MTZ enhanced contraction of burn wounds, but differs from previous study ${ }^{[4]}$ in which $20 \mathrm{mg} / \mathrm{kg}$, b.w., i.p., of MTZ failed to alter wound contraction The discrepancy may be because of the smaller dose used in contrast with the present dose of $108 \mathrm{mg} / \mathrm{kg}$, b.w., p.o. There is paucity of information about the influence of KCZ on wound healing.

The present finding of increased wound breaking strength in MTZ-treated animals differs from the earlier report ${ }^{[4]}$ in that MTZ did not alter the breaking strength of skin wounds in the dose of $20 \mathrm{mg} / \mathrm{kg}$, b.w., i.p., for 7 days. Smaller dose and shorter duration of treatment in the previous study $(108 \mathrm{mg} /$ $\mathrm{kg}$ for 10 days in the present study) may explain the discrepancy.

In the present study, MTZ, TNZ and FLZ significantly increased the healing of dead space wounds as evidenced by increased granulation tissue formation, granulation tissue breaking strength, and hydroxyproline content. There are scanty reports about the influence of FLZ and KCZ on dead space wounds. However, MTZ and TNZ in contrast to present findings, have been reported to suppress healing of dead space wounds. ${ }^{|3|}$ The discrepancy may be owing to the higher dose of MTZ (160 mg/kg/day) used in the earlier study in contrast to $(108 \mathrm{mg} / \mathrm{kg} /$ day) the dose used in the present study. It is difficult to explain the discrepancy of the effect of TNZ on healing process, because TNZ has been used in the same dose $(180 \mathrm{mg} / \mathrm{kg} /$ day) in both the studies.

The enhanced healing of dead space wounds by MTZ, TNZ and FLZ in the present study is further supported by histological studies of granulation tissue. There was an increased number of fibroblasts and collagen content in treated groups in contrast to control groups.

Prohealing effect of MTZ, TNZ and FLZ is unlikely to be due to their antimicrobial activity because the wounds were clean and healthy in the present study, though in infected wounds it could be a major contributing factor. The direct prohealing properties of MTZ has been attributed to its enhanced keratinization and epithelization, ${ }^{[14]}$ as well as antioxidant activity. ${ }^{[5]}$ Probably TNZ and FLZ may be sharing the above activities of MTZ, because they are chemically related. The prohealing mechanisms of these drugs need to be elucidated.

It is enigmatic to explain why KCZ has not significantly favoured healing process like other azoles used in the present study. However, its neutral effect on the healing process could be because of its ability to inhibit polymorphonuclear leucocyte chemotaxis, ${ }^{[15]}$ which has a considerable role in healing process.

In conclusion, perioperative use of MTZ, TNZ and FLZ could promote the healing of surgical wounds, if the present findings could be extrapolated to clinical situation, but again wellplanned clinical studies are needed.

\section{Acknowledgments}

The authors are grateful to the Principal, J.N. Medical College, Belgaum for providing facilities and Dr. P.R. Malur, Professor of Pathology for his guidance in microscopic studies. I also thank Mr. Mallapur, Biostatistician, Mr. A. V. Karvekar and Mr. M. D. Kankanwadi for their skilful assistance.

\section{References}

1. Khan M, Patil PA, Shobha JC. Influence of Bryophyllum pinnatum (Lam.) leaf extract on wound healing in albino rats. J Natural Remedies 2004;4:41-6.

2. Rathi B, Patil PA, Baheti AM. Evaluation of aqueous extract of pulp and seeds of Moringa oleifera for wound healing in albino rats. $J$ Natural Remedies 2004:4:145-9.

3. Prasad D, Rao CM. Wound healing profiles of Ketorolac, metronidazole and tinidazole administered post surgically. Indian J Expt Biol 1995;33:845-7.

4. Borden EB, Sammartano RJ, Dembe C, Boley SJ. The effect of metronidazole on wound healing in rats. Surgery 1985;97:331-5.

5. Rao CM, Ghosh A, Raghothama C, Bairy KL. Does metronidazole reduce lipid peroxidation in burn injuries to promote healing? Burns 2002;28:427-9.

6. Paget GE, Barnes JM, Toxicity tests. In : Laurence DR Bacharach AL, editors. Evaluation of drug activities Pharmacometrics. London and New York: Academic Press; 1964.

7. Ehrlich HP, Hunt TK. The effects of cortisone and anabolic steroids on the tensile strength of healing wounds. Ann Surg 1969;170:203-6.

8. Lee KH. Studies on the mechanism of action of salicylates II. Retardation of wound healing by aspirin. J Pharm Sci 1968;57:1042-3.

9. Morton JJ, Malone MH. Evaluation of vulnerary activity by an open procedure in rats. Arch Int Pharmacodyn Ther 1972;196:117-26.

10. Turner RA. 'Antiinflammatory agent' in screening methods of pharmacology. $2^{\text {nd }}$ ed. New York: Academic Press; 1965.

11. Dipasquale G, Meli A. Effect of body weight changes on the formation of cotton pellet-induced granuloma. J Pharm Pharmacol 1965;17:379-82.

12. Woessner JF. The determination of hydroxyproline in tissue samples. Arch Biochem 1961;93:440.

13. Conn $\mathrm{H}$, Nanda VS. Prophylactic fluconazole promotes reepithelization in fullface carbon dioxide laser skin resurfacing. Lasers Surg Med 2000;26:201-7.

14. Pandey S, Basheer M, Roy S, Udupa N. Development and evaluation of transdermal formulations containing metronidazole and norfloxacin for the treatment of burn wound. Indian J Expt Bio 1999;37:450-4.

15. Roman-Kelly B, Ferrante A, Thong YH. Modificatioin of polymorphonuclear leucocyte function by imidazoles. Int J Immunopharmacol 1984;6:389-93.

\section{Join "IndPharm" \\ IJP uses "IndPharm" to broadcast announcements. Want to join? Please E-mail: adithan@vsnl.com}

